\title{
ON SUBMANIFOLDS WITH HARMONIC MEAN CURVATURE
}

\author{
MANUEL BARROS AND OSCAR J. GARAY
}

(Communicated by Christopher Croke)

\begin{abstract}
The classification of curves in $E^{m}$ with harmonic mean curvature vector field in the normal bundle is obtained and then it is used to obtain some applications.
\end{abstract}

\section{INTRODUCTION}

Let $x: M^{n} \rightarrow E^{m}$ be an isometric immersion of a Riemannian manifold in the Euclidean space. Denote by $H$ and $\Delta$ the mean curvature vector field of $(M, x)$ and the Laplacian of $M$ respectively. A classical and well-known equation of Beltrami gives a nice relation between $H$ and $\Delta$, namely $\Delta x=$ $-n H$. Therefore minimal submanifolds in Euclidean space correspond with harmonic submanifolds.

Submanifolds satisfying $\Delta H=0$ were called biharmonic submanifolds by B. Y. Chen. He also conjectured that biharmonicity implies harmonicity (and so minimality). This conjecture has been proved to be true in some special cases. For instance, B. Y. Chen [Ch] solved the conjecture for surfaces in $E^{3}$. Also I. Dimitric [Di] did so for curves in $E^{m}$ by showing that straight lines in $E^{m}$ are the only biharmonic curves in $E^{m}$.

On the other hand if one considers a circle in $E^{2}$ or more generally a Cornu spiral in $E^{2}$, the curvature function $k$ is a linear function in terms of the arclength parameter. Then $k$ is harmonic and it automatically gives the harmonicity of $H$ in the normal bundle.

In this short note we classify curves in Euclidean space $E^{m}$ with harmonic mean curvature vector field in the normal bundle. Besides straight lines, circles and Cornu spirals (which correspond with planar curves) we will obtain a twoparameter family of curves living in 2-spheres of $E^{3}$ (see Theorem 1).

This result is used to get some applications. First we will get the complete classification of hypercylinders constructed on curves in $E^{p}$ with harmonic mean curvature vector field in the normal bundle, so obtaining an extension of Theorem 79 of [Ch-Ve].

In the last section we will use Theorem 1 jointly with the Hopf fibration

Received by the editors September 6, 1993 and, in revised form, January 11, 1994.

1991 Mathematics Subject Classification. Primary 53C40, 53A05.

Key words and phrases. Mean curvature function, mean curvature vector field, harmonic field. Research partially supported by a DGICYT grant No. PB91-0705-C02-01. 
$\Pi: S^{3} \rightarrow S^{2}$ to get a one-parameter family of surfaces in $S^{3}$ with non-constant harmonic mean curvature function.

\section{CuRves IN EUCLIDEAN SPACE WITH haRMonic MEAN CURVATURE VECTOR FIELD IN THE NORMAL BUNDLE}

Consider an immersed curve $\beta=\beta(s): I \subset R \rightarrow E^{m}$ where $s$ denotes the arclength parameter of $\beta . T=T(s)=\beta^{\prime}(s)$ will be the unit tangent vector field of $\beta$. Assume that $\beta$ is not a plane curve (it is not contained at any 2-plane of $E^{m}$ ) so we can define a 2-dimensional subbundle, say $\nu$, of the normal bundle $\Lambda$ of $\beta$ into $E^{m}$ as

$$
\nu(s)=\operatorname{Span}\left\{\xi_{2}, \xi_{3}\right\}(s)
$$

where $\xi_{2}$ and $\xi_{3}$ are unit normal vector fields to $\beta$ defined by

$$
\begin{gathered}
T^{\prime}(s)=k(s) \xi_{2}(s), \\
\xi_{2}^{\prime}=-k(s) T(s)-\tau(s) \xi_{3}(s)
\end{gathered}
$$

where $k>0$ is the curvature (the first curvature if $m>3$ ) and $\tau$ is the torsion (the second curvature with $\tau>0$ if $m>3$ ) of $\beta$.

Denote by $\nu^{\perp}$ the orthogonal complementary subbundle of $\nu$ in $\Lambda$. Certainly the fibers of $\nu^{\perp}$ have dimension $m-3$. Therefore the Frenet equations of $\beta$ can be partially written as:

$$
\begin{aligned}
& T^{\prime}(s)=k(s) \xi_{2}(s), \\
& \xi_{2}^{\prime}(s)=-k(s) T(s)-\tau(s) \xi_{3}(s), \\
& \xi_{3}^{\prime}(s)=\tau(s) \xi_{2}(s)+\delta(s)
\end{aligned}
$$

where $\delta(s) \in \nu^{\perp}(s)$ for all $s \in I$.

The curvature vector field of $\beta$ (the mean curvature vector field of $\beta$ ) is defined by

$$
H(s)=T^{\prime}(s)=k(s) \xi_{2}(s) .
$$

Equations (5) and (6) also give how the normal connection $D$ of $\beta$ into $E^{m}$ behaves on $\nu$

$$
\begin{aligned}
& D_{T} \xi_{2}=-\tau \xi_{3}, \\
& D_{T} \xi_{3}=\tau \xi_{2}+\delta .
\end{aligned}
$$

Let $\Delta^{D}$ be the Laplacian associated with $D$. One can use the Frenet equations, (8) and (9) to compute $\Delta^{D} H$ and so one obtains

$$
\Delta^{D} H=\left(-k^{\prime \prime}+k \tau^{2}\right) \xi_{2}+\left(2 k^{\prime} \tau+k \tau^{\prime}\right) \xi_{3}+k \tau \delta .
$$

Now let us consider that $\beta$ has harmonic mean curvature vector field in $\Lambda$, which means $\Delta^{D} H=0$ and so

$$
\begin{aligned}
k^{\prime \prime}(s)-k(s) \tau^{2}(s) & =0, \\
2 k^{\prime}(s) \tau(s)+k(s) \tau^{\prime}(s) & =0, \\
k(s) \tau(s) \delta(s) & =0 .
\end{aligned}
$$

Equation (12) gives $\tau(s) k^{2}(s)=c$, where $c$ is some constant which we may assume to be nonzero since $\beta$ is not a plane curve. Consequently (13) implies 
that $\delta$ must vanish identically. This means that $\nu$ (and so $\nu^{\perp}$ ) is a parallel subbundle in $\Lambda$. Furthermore $\nu^{\perp}$ is composed of totally geodesic directions, and consequently we can reduce codimension to some $E^{3}$ totally geodesic into $E^{m}$ with $\beta \subset E^{3}$. The normal bundle of $\beta$ into $E^{3}$ is essentially $\nu$.

After that we still have equations (11) and (12) reduced to

$$
\begin{aligned}
k^{\prime \prime} & =k \tau^{2}, \\
c & =\tau k^{2}
\end{aligned}
$$

and certainly we can use standard arguments to get the integration of these equations.

We begin by doing a first integration to obtain

$$
\left(k^{\prime}\right)^{2}=b-\frac{c^{2}}{k^{2}}
$$

where $b$ is some positive constant.

Now the general solution of (16) is given by

$$
k(s)=\sqrt{b(s-a)^{2}+\frac{c^{2}}{b}}
$$

where $a$ is a new constant.

Finally the torsion is given by

$$
\tau(s)=\frac{b c}{b^{2}(s-a)^{2}+c^{2}} .
$$

The fundamental theorem of curves says that there exists a curve in $E^{3}$ (unique up to motions) whose curvature and torsion functions are given by (17) and (18) respectively.

The parameter $a$ is not essential in the sense that it depends of the origin we use to measure the arclength function of $\beta$. Therefore the class of curves we have just obtained can be a priori parametrized into $R-\{0\} \times R^{+}$according to the values of $c$ and $b$ respectively. But from (18) the sign of $c$ is determined from the orientation of $\beta$. So essentially we have a family of curves in $E^{3}$ parametrized into $R^{+} \times R^{+}$.

Also notice that from (17) and (18) we get

$$
\frac{1}{k^{2}}+\frac{\left(k^{\prime}\right)^{2}}{\tau^{2} k^{4}}=\frac{b}{c}
$$

and this shows that these curves are spherical curves, namely, they are contained in 2-spheres of $E^{3}$ centered at the origin (it does not matter) and with radii $\sqrt{\frac{b}{c}}$. From now on we will denote this class of spherical curves by

$$
\Omega=\left\{\beta_{b c} \subset S^{2}\left(\sqrt{\frac{b}{c}}\right) \subset E^{3} /(c, b) \in R^{+} \times R^{+}\right\} .
$$

This situation can be summarized in the following:

Theorem 1. Let $\beta: I \subset R \rightarrow E^{m}$ be a full arclength parametrized curve; then it has harmonic mean curvature vector field in the normal bundle if and only if 
either:

(1) $m=2$ and $\beta$ is a straight line, or

(2) $m=2$ and $\beta$ is a circle, or

(3) $m=2$ and $\beta$ is a Cornu spiral, or

(4) $m=3$ and $\beta \in \Omega$.

Remark 1. To better understand the statement of the last theorem let us recall an easy classical fact which says that the straight lines, the circles and the Cornu spirals are the only plane curves with harmonic curvature functions. Theorem 1 should be compared with the classification of biharmonic curves in $E^{m}$ (see [Di]).

As a first application of the above theorem, we can obtain a nice generalization of Theorem 79 of [Ch-Ve]. In fact, we obtain the complete classification of hypercylinders constructed on curves in $E^{p}$ with harmonic mean curvature vector field, in particular, those hypercylinders with harmonic mean curvature function.

Corollary 1. The only hypercylinders $M^{p+1}$ in $E^{p+q}$ with harmonic mean curvature vector field in the normal bundle are open parts of the following submanifolds:

(1) $M=R^{p+1}$, or

(2) $M=R^{p} \times \beta, \beta$ being a circle, or

(3) $M=R^{p} \times \beta, \beta$ being a Cornu spiral, or

(4) $M=R^{p} \times \beta_{b c}$, for any pair of positive numbers $b, c$.

3. A CLASS OF SURFACES IN $S^{3}$

WITH NONCONSTANT HARMONIC MEAN CURVATURE

In this section and without loss of generality we will consider the unit 3-sphere in $E^{4}$ and the Hopf fibration $\Pi: S^{3} \rightarrow S^{2}$ on the unit 2-sphere in $E^{3}$.

Given a curve $\beta: I \subset R \rightarrow S^{2} \subset E^{3}$ with curvature and torsion functions $k>0$ and $\tau$ respectively, we have

$$
\frac{1}{k^{2}}+\frac{\left(k^{\prime}\right)^{2}}{k^{4} \tau^{2}}=1
$$

The curvature of $\beta$ into $S^{2}$ is given by

$$
\rho(s)=\frac{k^{\prime}(s)}{k(s) \tau(s)} .
$$

Lemma 1. For any positive number $b$, the curve $\beta_{b}=\beta_{b b}$ has harmonic curvature function in the unit 2-sphere $S^{2} \subset E^{3}$.

Proof. Just use (17) and (18) with $b=c$ to see that the curvature function of $\beta_{b}$ into the unit sphere, say $\rho_{b}$, is a linear function. Q.E.D.

Given an immersed curve $\beta: I \subset R \rightarrow S^{2}$, choose $\eta: I \subset R \rightarrow S^{3}$ such that $\Pi \circ \beta=\eta$. We will assume that it is parametrized by arclength and cuts the fibres of $\Pi$ orthogonally. With the notation $S^{1}:=R / 2 \pi Z$ we define an immersion $X$ of the cylinder $I \times S^{1}$ into $S^{3}$ by

$$
X(s, \varphi)=e^{i \varphi} \eta(s) .
$$


$X$ is called the Hopf cylinder corresponding to the curve $\beta$. By a direct computation, we can obtain the shape operator $A$ to $X(s, \varphi)$

$$
A(s, \varphi)=\left(\begin{array}{cc}
2 \rho(s) & 1 \\
1 & 0
\end{array}\right)
$$

Theorem 2. For any positive number $b$, the Hopf cylinder $M_{b}=\Pi^{-1}\left(\beta_{b}\right)$ has nonconstant harmonic mean curvature function in $S^{3}$. Moreover, given a Hopf cylinder $M=\Pi^{-1}(\beta)$ in $S^{3}$, then it has harmonic mean curvature function if and only if either

(1) $\beta$ is a great circle in $S^{2}$ and $M$ is the Clifford torus, or

(2) $\beta$ is a small circle in $S^{2}$ and $M$ is a rectangular tori, or

(3) $\beta=\beta_{b}$ in $S^{2}$ and $M=M_{b}$ for some positive number $b$.

Proof. From (24), the mean curvature of $M=\Pi^{-1}(\beta)$ in $S^{3}$ coincides with the curvature of $\beta$ in $S^{2}$. The Laplacian of $M$ in the $X(s, \varphi)$ parametrization is given by

$$
\Delta=-\left(\frac{\partial^{2}}{\partial s^{2}}+\frac{\partial^{2}}{\partial \varphi^{2}}\right) .
$$

Now the first part of the theorem is clear. To see the "if" part of the second statement notice that cases (1) and (2) correspond with the constancy for the mean curvature. Finally to prove the "only if" part of the second statement just say that a curve with harmonic curvature in $S^{2}$ must belong to $\Omega$. Q.E.D.

\section{REFERENCES}

[Ch] B. Y. Chen, Some open problems and conjectures on submanifoldss of finite type, Soochow J. Math. 17 (1991), 169-188.

[Ch-Ve] B. Y. Chen and L. Verstraelen, Differential geometry of Laplace transformations of submanifolds, Geometry and Topology of Submanifolds IV, World Scientific, Singapore and Teaneck, NJ, 1992, pp. 51-73.

[Di] I. Dimitric, Submanifolds of $E^{m}$ with harmonic mean curvature vector, Bull. Inst. Math. Acad. Sinica 20 (1992), 53-65.

[Pi] U. Pinkall, Hopf tori in $S^{3}$, Invent. Math. 81 (1985), 379-386.

Departamento de Geometria y Topologia, Facultad de Ciencias, Universidad de Granada, 18071 Granada, Spain

E-mail address: garay@ugr.es 